Journal of Computer Science 7 (10): 1525-1531, 2011

ISSN 1549-3636

(C) 2011 Science Publications

\title{
Design and Cost Performance of Decoding Technique for Hybrid Subcarrier Spectral Amplitude Coding-Optical Code Division Multiple Access System
}

\author{
R.K.Z. Sahbudin, M.K. Abdullah, M. Mokhtar, S. Hitam and S.B. Ahmad Anas \\ Department of Computer and Communication Engineering, \\ Faculty of Engineering, Universiti Putra Malaysia, \\ 43400, Serdang, Selangor, Malaysia
}

\begin{abstract}
Problem statement: A hybrid technique combining subcarrier multiplexed and Spectral Amplitude Coding-Optical Code Division Multiple Access (SAC-OCDMA) deploying the KhazaniSyed code was proposed. It was proposed as a mean of increasing the maximum number of simultaneous active users by increasing the subcarrier and/or the SAC-OCDMA code word. Multiple Access Interference (MAI) is the most dominant cause of performance degradation in SACOCDMA systems. Approach: A decoding technique which was simple and easy to build named Spectral Direct Decoding (SDD) technique was proposed for the hybrid system of subcarrier SACOCDMA. Results: The comparison between the complementary subtraction and proposed SDD techniques for hybrid subcarrier SAC-OCDMA system in terms of cost performance was presented here. Results showed that the cost performance of the SDD technique offers a significant improvement over the complementary subtraction detection technique. Conclusion: It is shown that the cost performance of the hybrid system using SDD technique is significantly better than using the Complementary subtraction technique.
\end{abstract}

Key words: Cost performance, SDD technique, hybrid subcarrier, Spectral Amplitude CodingOptical Code Division Multiple Access (SAC-OCDMA), Spectral Direct Decoding (SDD), communication systems, decoding technique

\section{INTRODUCTION}

The tremendous growth of the Internet and the World Wide Web, has made telecommunications one of the leading growth sectors in the world economy. Thus, the infrastructures need to be able to meet the increasingly high user's expectation. The vast bandwidth of the optical fiber enables a large number of simultaneous communication channels to be carried by a single optical fiber by any one of a number of multiplexing techniques. Therefore, in this study, a hybrid technique between subcarrier multiplexed and SAC-OCDMA deploying the Khazani-Syed (KS) (Sahbudin et al., 2009) code is proposed. It is proposed as a means of increasing the maximum number of simultaneous active users by increasing the subcarrier and/or the SAC-OCDMA codeword. Thus the resulting hybrid system is robust against interference, possesses high transmission security and increases the channel capacity in the existing optical fibers.

The SAC-OCDMA systems with good design of the code sequences and detection or decoding technique were proposed as a means of increasing the maximum number of simultaneous active users and reduce the effect of Multiple Access Interference (MAI) (AbRahman et al., 2009; Al-Galbi et al., 2009) at the same time. MAI is the main cause of performance degradation in SAC-OCDMA systems. The effect of MAI can be eliminated by using Complementary subtraction technique, which is also known as balanced detection technique (Tsai et al., 2009) if the spectral amplitude is flat across the whole spectrum. However, in practical, this does not happen and it will still cause MAI. Consequently, it is extremely important to design decoding technique in such a way that MAI is minimized regardless of the number of users and

Corresponding Author: Ratna Kalos Zakiah Sahbudin Department of Computer and Communication System Engineering, Faculty of Engineering, Universiti Putra Malaysia, 43400 Serdang, Selangor, Malaysia Tel: 603-89466431 Fax: 603-86567127 
maintain the performance of the system at a minimal cost. Alternatively, the subcarrier multiplexed technology is simple and cost-effective. (Kosek et al., 2007). It provides a way to take advantage of the huge bandwidth of the optical fiber using well-known microwave techniques for which components are established and commercially available (Kourtessis et al., 2007).

Researches Yang (2006) and Huang and Chang (2006) use Complementary method at the receiver side to recover the original signal. A decoding technique which is simple and easy to build named SDD technique is proposed for the hybrid system of subcarrier SAC-OCDMA. This technique reduces the receiver complexity, cost and at the same time improves the system performance. In this study, we present the design and cost performance between the Complementary subtraction and SDD decoding techniques for the hybrid subcarrier SACOCDMA system.

Hybrid subcarrier SAC-OCDMA system: The block diagram of the hybrid subcarrier SAC-OCDMA system is shown in Fig. 1. At the transmitter, the data (user) is mixed with a different microwave carrier frequency $f_{\mathrm{i}}$.

The subcarriers are combined and optically modulated onto the code sequence $c_{i}$, using an Mach Zehnder Optical External Modulator (OEM). Then m modulated code sequences are combined together via the optical combiner and transmitted through the optical fiber. So in this hybrid system, each user is assigned with a particular code sequence $c_{i}$ and subcarrier frequency $f_{i}$, where the pair of $\left(c_{i}, f_{i}\right)$ is unique with respect to every other user. At the receiver, an optical splitter is used to separate the different modulated code sequences. Only the intended receiver is able to correctly demodulate the detected signal, made possible by using a matched code sequence and the unmatched signals are filtered out. Then, the photodetector is used to detect the decoded signal. A splitter and an electrical Band Pass Filter (BPF) are used to split the subcarrier multiplexed signals and to reject the unwanted signals, respectively. In order to recover the original transmitted data, the incoming signal is electrically mixed with a local microwave frequency $f_{i}$ and filtered using LowPass Filter (LPF). Hence, the proposed hybrid system has a high transmission security since each receiver must tune to the correct pair of $\left(c_{i}, f_{i}\right)$ to receive the desired data.

In this study, optical bandpass filters are used as the encoders and decoders that are discussed. The number of filters needed is equal to the number of 'ones' in the code sequence. Assumption is made here that separate filters are used for different chip wavelength, as been widely proposed in SAC-OCDMA implementation (Anas et al., 2009).

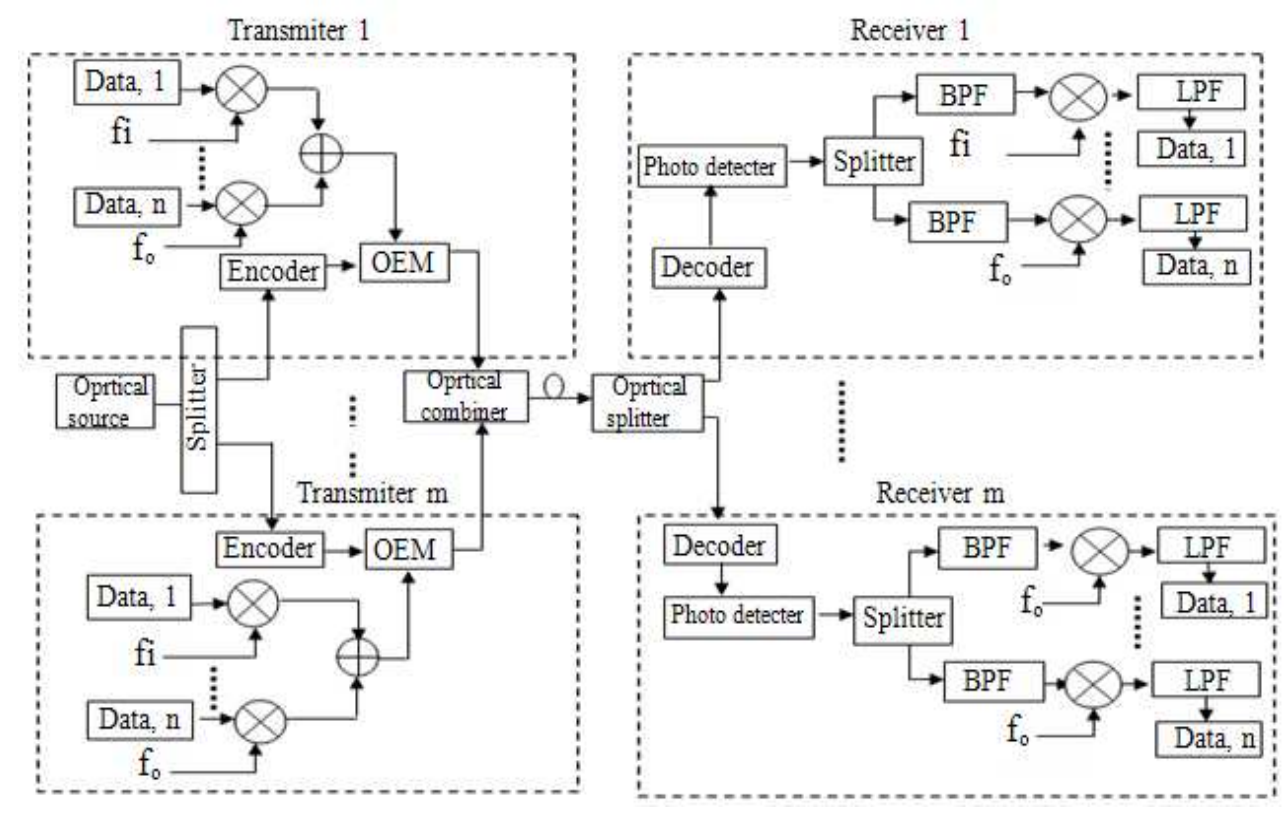

Fig. 1: Block diagram of subcarrier SAC-OCDMA system 
In the KS code, two chips (wavelengths) always occur next to each other, while in other codes published Prucnal (2006), two adjacent wavelengths are separated. Since chip 'ones' always occurs in pairs, one filter of a larger bandwidth (doubled) could be used instead of using two filters to represent two distinct 'ones'. Hence, the number of filters at the encoder and decoder for a system employing KS code is reduced by half (Sahbudin and Abdullah, 2009).

\section{MATERIALS AND METHODS}

Complementary Subtraction Detection Technique: For example, let us consider the KS code sequences as shown in Table 1.

Note that $\lambda_{\mathrm{i}}$ where $\mathrm{i}$ is $1,2, \ldots \mathrm{N}$, represents the spectral position of the chips in the KS code sequence.

The implementation of a hybrid subcarrier SACOCDMA system using Complementary subtraction technique is shown in Fig. 2.

The optical pulses are encoded according to the KS code sequences denoted as $\mathrm{X}=(1100)$ and $\mathrm{Y}=(0110)$. The subcarrier signal is then optically modulated with the KS code sequence. The outputs of the two Mach Zehnder External Modulators (OEM) are combined and transmitted through an optical fiber. As shown in Receiver 1, the signals are split and decoded separately by two Complementary decoders. The outputs from the Complementary filters are detected by the two photo detectors (PIN) connected to a subtractor. In order to decode the KS code and detect the signal, this technique requires five filters, four photodetectors and two subtractors. Three filters with the bandwidth twice the chip width for 'ones' at the position of $\lambda_{1}$ and $\lambda_{2}, \lambda_{3}$ and $\lambda_{4}, \lambda_{2}$ and $\lambda_{3}$ and two separate filters for $\lambda_{1}$ and $\lambda_{4}$. To eliminate the MAI from undesired users, an attenuator is required at PIN 2 and PIN 4. This is explained below.

Let $\mathrm{C}_{\mathrm{k}}(\mathrm{i})$ denote the ith element of the kth KS code sequence with $\overline{\mathrm{C}}_{1}(\mathrm{i})$ as its complement. The code properties for the Complementary detection technique can be written as (Sahbudin and Abdullah, 2009):

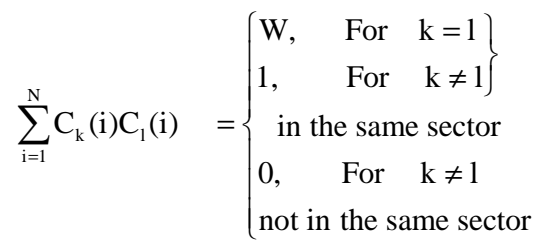

Table 1: Example of $\mathrm{KS}$ code with weight, $\mathrm{W}=2$

\begin{tabular}{lllll}
\hline \multicolumn{5}{c}{ Code sequence } \\
\hline & 11 & 12 & 13 & 14 \\
$\mathrm{X}$ & 1 & 1 & 0 & 0 \\
$\mathrm{Y}$ & 0 & 1 & 1 & 0 \\
\hline
\end{tabular}

And:

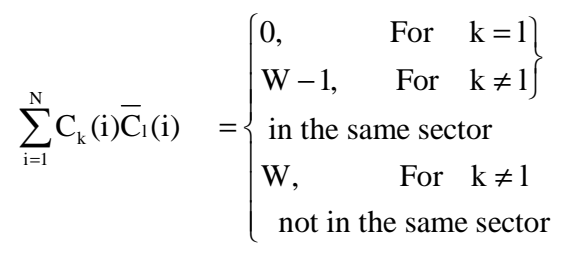

The condition of $\mathrm{k}$ and $\mathrm{l}$ in the same sector meaning that both code sequences are in $\mathrm{C}(1), \mathrm{C}(2)$ or $\mathrm{C}(\mathrm{M})$ as shown in Eq. 3. For the condition of $\mathrm{k}$ and 1 not in the same sector meaning that one of the code sequences might be in $\mathrm{C}(1)$ and the other code sequence is in $\mathrm{C}(2)$ or $\mathrm{C}(\mathrm{M})$.

$\mathrm{C}(\mathrm{m})=\left[\begin{array}{cccc}\mathrm{C}(1) & 0 & 0 & 0 \\ 0 & \mathrm{C}(2) & 0 & 0 \\ 0 & 0 & \ddots & 0 \\ 0 & 0 & 0 & \mathrm{C}(\mathrm{M})\end{array}\right]$

The matrix size of each 0 and $\mathrm{C}(\mathrm{m}), 1<\mathrm{m} \leq \mathrm{M}$ is the same as $\mathrm{C}(1)$ matrix size. For a fixed value of weight, when the number of mapping is increased, the code size is extended by $\left(\mathrm{m} \times \mathrm{K}_{\mathrm{B}}\right)$ and the basic code is extended diagonally as shown in Eq. 3. $\mathrm{K}_{\mathrm{B}}$ is given by (Sahbudin and Abdullah, 2009):

$\mathrm{K}_{\mathrm{B}}=\frac{\mathrm{W}}{2}+1$

In order to eliminate the MAI, the complement cross correlation $\mathrm{C}_{k}(\mathrm{i}) \overline{\mathrm{C}}_{1}(\mathrm{i})$ must be multiplied by an attenuator with a transmission coefficient, $\mathbf{T}$ so that the cross correlation $C_{k}(i) \bar{C}_{1}(i)$ subtracted from $C_{k}(i) C_{1}(i)$ is equal to zero. Therefore, the subtraction can be written as:

$$
\begin{aligned}
& \sum_{\mathrm{i}=1}^{\mathrm{N}} \mathrm{C}_{\mathrm{k}}(\mathrm{i}) \mathrm{C}_{1}(\mathrm{i})-\mathrm{T} \sum_{\mathrm{i}=1}^{\mathrm{N}} \mathrm{C}_{\mathrm{k}}(\mathrm{i}) \overline{\mathrm{C}}_{1}(\mathrm{i}) \\
& = \begin{cases}\mathrm{W}, & \mathrm{k}=1 \\
0, & \mathrm{k} \neq 1\end{cases}
\end{aligned}
$$

Where:

$$
\mathrm{T}=\frac{1}{\left[(\mathrm{~W}-1)+\frac{\mathrm{WK}_{\mathrm{B}}(\mathrm{m}-1)}{\left(\mathrm{K}_{\mathrm{B}}-1\right)}\right]}
$$




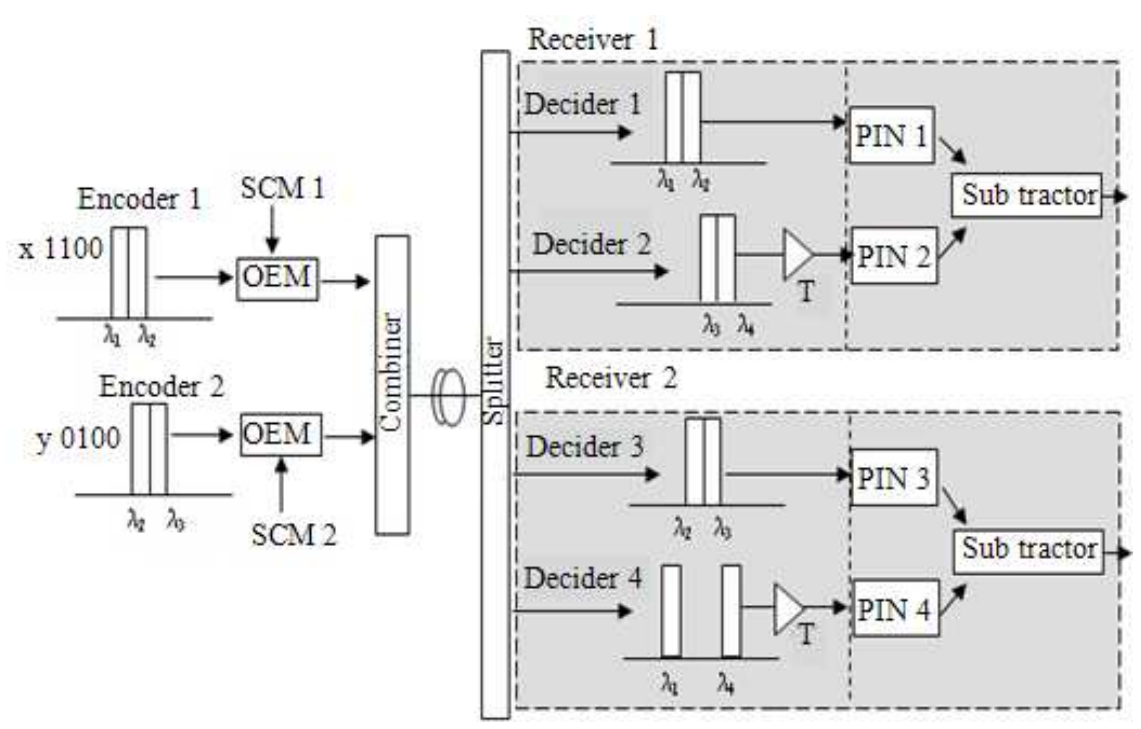

Fig. 2: Hybrid system using Complementary subcarrier SAC-OCDMA subtraction technique

Eq. 6 is when the number of mapping is greater than 1 . Without the mapping or $\mathrm{m}=1$, Eq. 6 becomes:

$$
\mathrm{T}=\frac{1}{[(\mathrm{~W}-1)]}
$$

Thus for $\mathrm{W} \gg>1$, Eq. 7 becomes:

$$
\mathrm{T}=\frac{1}{\mathrm{~W}}
$$

Eq. 5 shows that when the code sequence is matched, the weight $\mathrm{W}$ is obtained. However when the code sequences are unmatched, the weight is zero, meaning that the MAI is eliminated in this system.

Spectral Direct Decoding (SDD) technique: The hybrid subcarrier SAC-OCDMA system using SDD technique is shown in Fig. 3. For two KS code sequences as shown in Fig. 3, it requires only two filters for the decoders, which are used to filter the non-overlapping chips, $\lambda_{1}$ and $\lambda_{3}$ because the overlapping chips of the two code sequences may cause interference at the receiver (Khaleghi et al., 2010). The number of photodetectors is reduced to two. It does not require any subtractor and attenuator at the receiver. Note that it is assumed that the power per chip is sufficient for the data recovery.

If $\mathrm{C}_{k}(\mathrm{i})$ denotes the ith element of the kth KS code sequence, the code properties for SDD technique can be written as:

$$
\sum_{i=1}^{N} C_{k}(i) C_{1}(i)=\left\{\begin{array}{lll}
\frac{W}{2}, & \text { For } & k=1 \\
0, & \text { For } & k \neq 1
\end{array}\right.
$$

When the code sequences are unmatched, the weight is zero, meaning that the MAI can be eliminated using the SDD technique.

In the analysis, the light source of each user is assumed to be unpolarized and has a flat spectrum over bandwidth $\Delta \mathrm{v} \mathrm{Hz}$ with magnitude $\mathrm{P}_{\mathrm{sr}} / \Delta \mathrm{v}$, where $\mathrm{P}_{\mathrm{sr}}$ is the effective power from the source at the decoder. The desired signal for this technique is:

$\mathbf{I}=\mathfrak{R}\left[\frac{\mathrm{P}_{\mathrm{sr}}}{\mathrm{N}}\right] \cdot\left[\frac{\mathrm{W}}{2} \mathrm{~d}_{1}\right]$

$\mathrm{I}=\frac{\Re P_{\mathrm{sr}} \mathrm{W}}{\mathrm{N} 2} \mathrm{~m}_{\mathrm{n}, \mathrm{k}} \mathrm{u}_{\mathrm{n}, \mathrm{k}}(\mathrm{t})$

$\mathrm{N}$ is the KS code length, $\Re$ is the responsivity of the photodetectors and $\mathrm{u}_{\mathrm{n}, \mathrm{k}}(\mathrm{t})$ is the normalized digital signal at the nth subcarrier channel of the kth code, where 0 and 1 are represented as digital signal " 0 " and "1", respectively. $\mathrm{m}_{\mathrm{n}, \mathrm{k}}$ is the modulation index of the nth subcarrier of the kth code. Assuming an identical modulation index for all subcarrier channels, it is necessary that $0<\mathrm{m}_{\mathrm{n}, \mathrm{k}} \leq \frac{1}{\mathrm{~N}_{\mathrm{c}}}$ (Stern et al., 2008).

Since only the non-overlapping chip is filtered through for SDD technique, PIIN noise is eliminated thus is not considered. PIIN noise occurs only when more than one optical signal is incident on the photodetector surface (Rad and Salehi, 2006; Capman, et al., 2006). 


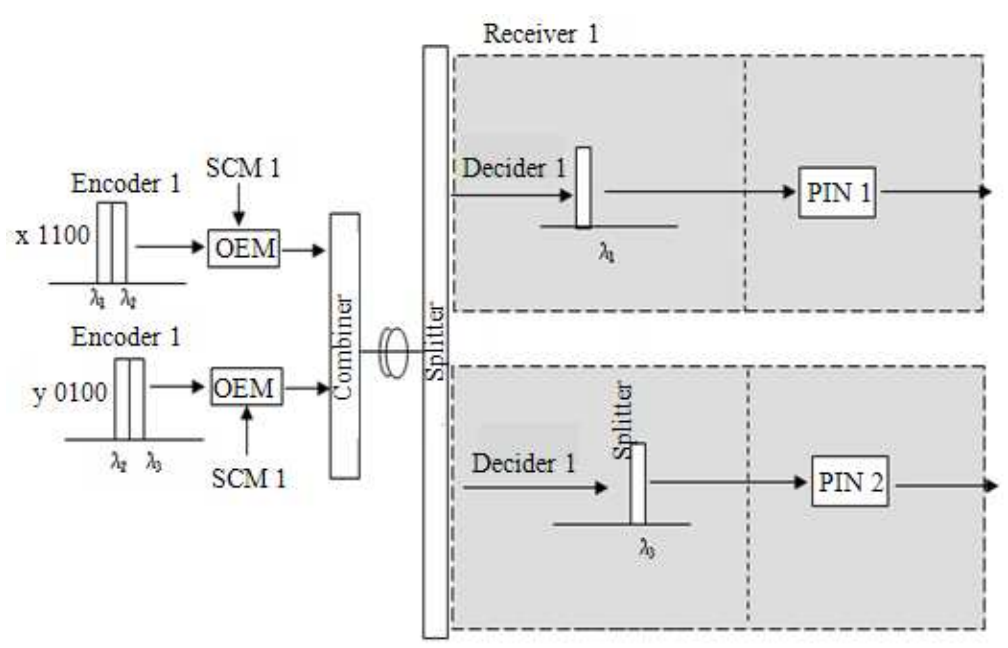

Fig. 3: Hybrid subcarrier SAC-OCDMA system using SDD technique

The total noise here is considered to be only the sum of shot noise, thermal noise and inter-modulation distortion noise as shown in Eq. 12, respectively.

$$
\begin{aligned}
& \left\langle\mathrm{I}^{2}\right\rangle=\mathrm{eB} \boldsymbol{R} \cdot\left[\frac{\mathrm{P}_{\mathrm{sr}}}{\mathrm{N}}\left(\frac{\mathrm{W}}{2}\right)\right]+\frac{4 \mathrm{~K}_{\mathrm{b}} \mathrm{T}_{\mathrm{n}} \mathrm{B}}{\mathrm{R}_{\mathrm{L}}} \\
& +\mathrm{P}_{\mathrm{sr}}{ }^{2} \boldsymbol{R}^{2} \mathrm{~m}_{\mathrm{n}, \mathrm{k}}{ }^{6}\left[\frac{\mathrm{D}_{111}}{32}+\frac{\mathrm{D}_{21}}{128}\right]
\end{aligned}
$$

where, $D_{111}$ and $D_{21}$ are the number of inter-modulation terms of the type $f_{i}+f_{k}-f_{l}$ and $2 f_{i}-f_{k}$ falling on the nth subcarrier with $\mathrm{N}_{\mathrm{c}}$ number of subcarrier channels, respectively. They are given as (Al-Raweshidy and Komaki, 2002):

$$
\begin{aligned}
& \mathrm{D}_{111}=\frac{\mathrm{n}}{2}\left(\mathrm{~N}_{\mathrm{c}}-\mathrm{n}+1\right)+\frac{1}{4}\left[\left(\mathrm{~N}_{\mathrm{c}}-3\right)^{2}-5\right] \\
& -\frac{1}{8}\left[1-(-1)^{\mathrm{N}_{\mathrm{c}}}\right](-1)^{\mathrm{N}_{\mathrm{c}}+\mathrm{n}} \\
& \mathrm{D}_{21}=\frac{1}{2}\left[\mathrm{~N}_{\mathrm{c}}-2-\frac{1}{2}\left\{1-(-1)^{\mathrm{N}_{\mathrm{c}}}\right\}(-1)^{\mathrm{n}}\right]
\end{aligned}
$$

The SNR of the hybrid system using the SDD technique can be expressed as:

$$
\operatorname{SNR}=\frac{\left(\mathrm{Idd}_{\mathrm{d}}\right)^{2}}{\left\langle 1^{2}\right\rangle}
$$

$$
\mathrm{SNR}=\frac{\frac{\Re P_{\mathrm{str}}\left(\frac{\mathrm{W}}{2}\right)^{2} m_{\mathrm{n}, \mathrm{k}}^{2}}{\mathrm{~N}^{2}}}{\frac{\mathrm{e}^{2} \mathrm{~B} \mathrm{P}_{\mathrm{sr}}}{\mathrm{N}}\left[\frac{\mathrm{W}}{2}\right]+\frac{4 \mathrm{~K}_{\mathrm{b}} \mathrm{T}_{\mathrm{n}} \mathrm{B}}{\mathrm{R}_{\mathrm{L}}}+\mathrm{P}_{\mathrm{st}}^{2} \Re^{2} \mathrm{R}_{\mathrm{n}, \mathrm{k}}^{6}\left[\frac{\mathrm{D}_{111}}{32}+\frac{\mathrm{D}_{21}}{128}\right]}
$$

\section{RESULTS AND DISCUSSION}

The design of Complementary subtraction and SDD techniques has been described. It is obvious that the advantage of SDD is that fewer numbers of filters are required for the decoders. The number of filters that is needed in the decoders depends on the code weight, $\mathrm{W}$ and the basic code's row size or the basic number of codes, $\mathrm{K}_{\mathrm{B}}$. Eqs. 17 and 18 show the number of filters that are required at the decoder for the Complementary subtraction and SDD technique, respectively.

$\mathrm{F}_{\text {Comp }}=2 \mathrm{~K}_{\mathrm{B}}^{2}-\mathrm{K}_{\mathrm{B}-} 2$

$\mathrm{F}_{\mathrm{SDD}}=\mathrm{K}_{\mathrm{B}}^{2}-\mathrm{K}_{\mathrm{B}}$

Moreover SDD technique does not require subtractor and attenuator to eliminate MAI. Therefore, the use of fewer components to implement the SDD technique leads to a substantial cost reduction of the overall system.

Figure 4 clearly shows that the SDD technique requires fewer filters at the decoders than the Complementary subtraction technique as the number of weight increases. For an example, for weight equals to 10, SDD and Complementary subtraction techniques require 30 and 64 filters, respectively.

Figure 5-6 show the number of filters and costs required for the implementation of both techniques as the number of codes increases, respectively. The weight $\mathrm{W}$ is set at 4 . The costs are calculated base on the number of filters and attenuators that are required for the decoders. It does not take into account other components such as the encoders, optical sources and fibers, which are considered as similar to both of the systems. 


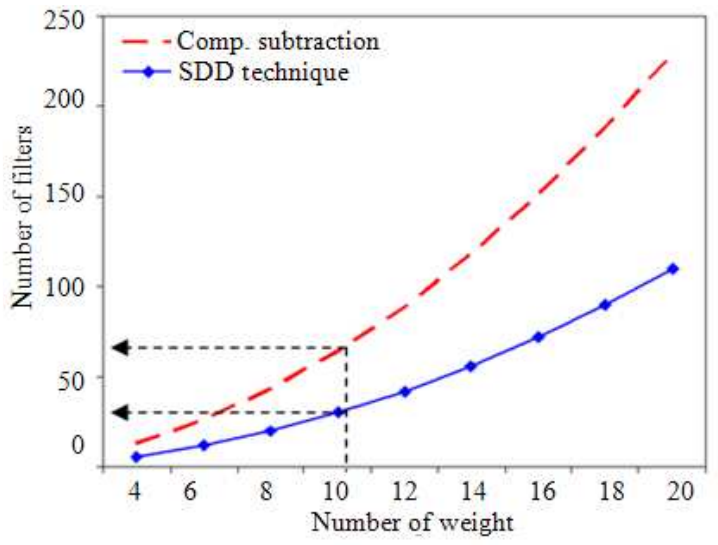

Fig. 4: Number of filters required for Complementary subtraction and SDD techniques as the code weight increases

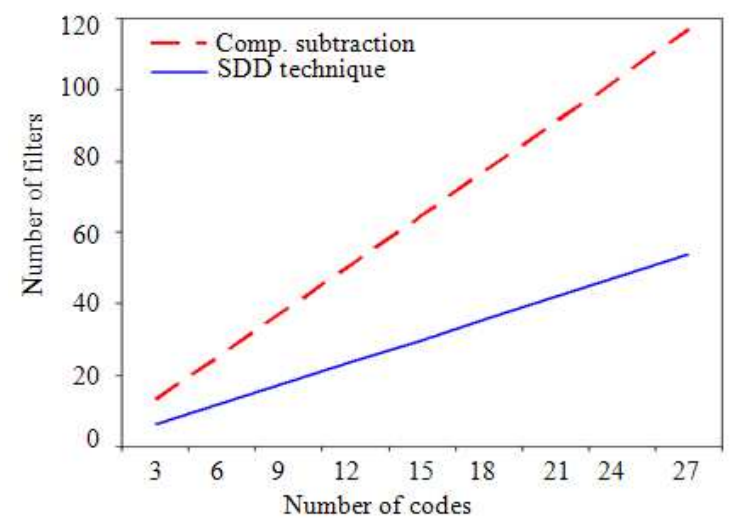

Fig. 5: Number of filters required for Complementary subtraction and SDD techniques as the number of codes increases

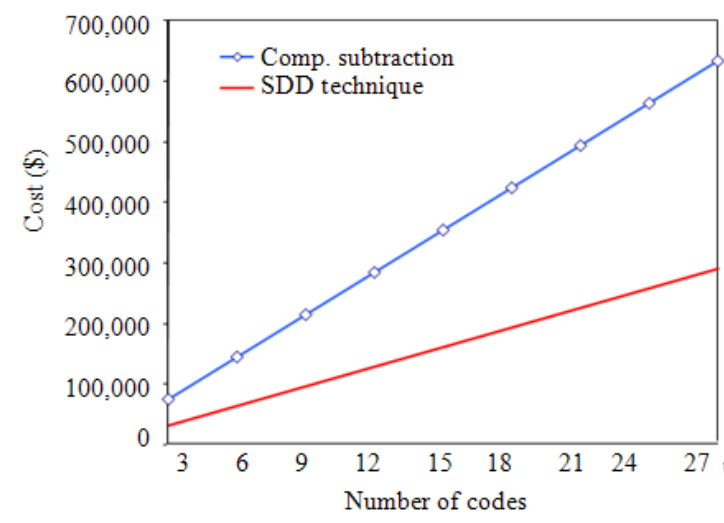

Fig. 6: Cost (\$) required for Complementary subtraction and SDD techniques as the number of codes increases

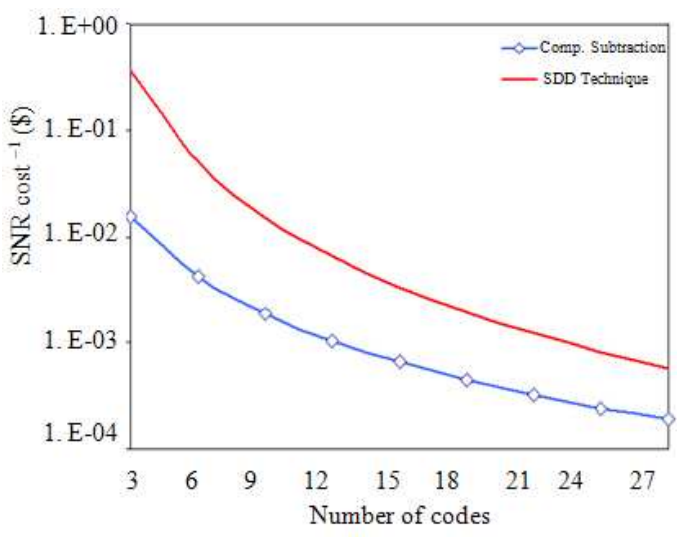

Fig. 7: Performance SNR/Cost ratio for hybrid system using Complementary subtraction and SDD techniques as the number of codes increases

In the economical evaluations, the cost for commercially available tunable optical filter and attenuator that has been provided by the manufacturer is $\$ 5366$ and $\$ 2590$, respectively. It is obvious that the SDD technique requires fewer filters than that using the Complementary subtraction as the number of codes increases, thus leads to a substantial reduction in cost of the overall hybrid system. Furthermore, it does not require attenuator to eliminate the MAI.

Figure 7 shows the performance signal-to-noise ratio (SNR)/cost ratio for the hybrid system employing the two decoding techniques. The performance SNR/cost ratio is defined as the SNR divided by the total price of all filters and attenuators. As larger SNR and/or lower cost are desirable, the larger the ratio the better. This is a new parameter proposed to represent the performancecost relationship for the hybrid subcarrier SAC-OCDMA system. The performance SNR/cost ratio for the hybrid system using SDD technique has been found to be greater than that using the Complementary subtraction technique as the number of codes increases, as shown in Fig. 7. It shows that theoretically it is advantageous to use the SDD technique.

\section{CONCLUSION}

The design and cost performance of decoding technique for the hybrid subcarrier SAC-OCDMA system have been presented in this study. It is shown that with the reduced number of filters and photodetectors required for the SDD technique, the complexity of the overall hybrid system is also reduced. It has been proven that the cost performance of the hybrid system using the SDD technique is improved significantly. The proposed hybrid system offers a great potential as a flexible and powerful technique as it can accommodate more users to access the network simultaneously. 


\section{ACKNOWLEDGEMENT}

This researchers is supported by UPM RUGS grant (Vote no. 91985).

\section{REFERENCES}

Sahbudin, R. K. Z. and M. K. Abdullah, 2009. Performance improvement of hybrid subcarrier multiplexing optical spectrum code division multiplexing system using spectral direct decoding detection technique. J. Optical Fiber Tech., 15: 266-273. 10.1016/J.YOFTE.2008.12.003

Ab-Rahman, M.S., B. Ng, N.M. Ibrahim and S. Shaari, 2009. Low-cost encoding device for optical code division multiple access system. Am. J. Eng. Applied Sci., 2: 317-323. DOI: 10.3844/AJEASSP.2009.317.323

Al-galbi, W. J.M. M. Mokhtar, A. F. Abas, S. B. A. Anas and R. K. Z. Sahbudin, 2009. Solving the near-far problem in dynamic frequency hoppingoptical code division multiple access using power control. J. Comput. Sci., 5: 413-418. DOI: 10.3844/JCSSP.2009.413.418

Tsai, C-M., Huang, J-F, 2009. Reduction of leakage effect with circular-free fiber-gratings codec array in OCDMA network. J. Optical Fiber Tech., 15: 445-450. DOI: 10.1016/J.YOFTE.2009.07.005

Kosek, H., Y. He, X. Gu, X. N. Fernando, 2007. Alloptical demultiplexing of WLAN and cellular CDMA radio signals. IEEE J. Lightwave Tech., 25: 1401-1409. DOI: 10.1109/JLT.2007.896767

Kourtessis, P. and S.D. Walker, 2007. A complete 8GHz QPSK-modem featuring novel subcarrier and data synchronization for optical communications. IEEE Trans. Commu., 55: 987- 995. DOI: 10.1109/TCOMM.2007.896048

Yang, C.-C, 2006. Modified legendre sequences for optical CDMA-based passive optical networks. IEEE Commu. Lett., 10: 393-395. DOI: 10.1109/LCOMM.2006.1633334
Huang, J.F. and Y.-T. Chang, 2006. Incoherent hybrid spectral polarization and amplitude coding implemented with specified orthogonal ternary code over balanced photo-detectors. In proceeding of the 4th Annual Communication Networks and Services Research Conference (CNSR'06), 24-25 May 2006, pp: 42-52. DOI: 10.1109/CNSR.2006.33

Ahmad Anas S.B., M.K. Abdullah, M. Mokhtar, S.A. Aljunid and S.D. Walker, 2009. Optical domain service differentiation using spectral-amplitudecoding. J. Optical Fiber Tech., 15: 26-32. DOI: 10.1016/J.YOFTE.2008.04.001

Prucnal, P. R., 2006. Optical code division multiple access: fundamentals and applications. CRC Taylor and Francis, pp. 199. ISBN: 084933683X, 9780849336836

Khaleghi, S. and M.R. Pakravan, 2010. Quality of service provisioning in Optical CDMA packet networks. J. Opt. Commun. Netw. 2: 283-292. DOI: $10.1364 / \mathrm{JOCN} .2 .000283$

Stern, T.E., G. Ellinas, K. Bala, 2008. Multiwavelength optical networks: architectures, design, and control Cambridge University Press, pp. 71. ISBN: $0521881390,9780521881395$.

Rad, M.M. and J.A. Salehi, 2006. Phase-induced intensity noise in digital incoherent all-optical tapped-delay line systems. J. Lightwave Tech., 24: 3059-3072. DOI: 10.1109/JLT.2006.878057

Capmany, J., B. Ortega and D. Pastor, 2006. A tutorial on microwave photonic filters. J. Light wave Tech., 24: 201-229.

Al-Raweshidy, H. and S. Komaki, 2002. Radio over Fiber Technologies for Mobile Communications Networks, Artech House, Inc. 